\title{
Generalized Analytical Solutions for Nonlinear Positive-Negative Index Couplers
}

\author{
Zh. Kudyshev, ${ }^{1,2}$ G. Venugopal, ${ }^{2}$ and N. M. Litchinitser ${ }^{2}$ \\ ${ }^{1}$ Department of Physics, Al-Farabi Kazakh National University, Almaty 050038, Kazakhstan \\ ${ }^{2}$ Department of Electrical Engineering, University at Buffalo, The State University of New York, Buffalo, NY 14260, USA
}

Correspondence should be addressed to N. M. Litchinitser, natashal@buffalo.edu

Received 9 January 2012; Accepted 17 February 2012

Academic Editor: Vladimir I. Gavrilenko

Copyright (C 2012 Zh. Kudyshev et al. This is an open access article distributed under the Creative Commons Attribution License, which permits unrestricted use, distribution, and reproduction in any medium, provided the original work is properly cited.

We find and analyze a generalized analytical solution for nonlinear wave propagation in waveguide couplers with opposite signs of the linear refractive index, nonzero phase mismatch between the channels, and arbitrary nonlinear coefficients.

\section{Introduction}

By leveraging the capabilities of photonics (speed) and of electronics (compactness) it may be possible to realize high-performance integrated optoelectronic systems for high bandwidth signal processing or sensing applications. Such integration requires the availability of ultracompact, ultrafast, low-loss components that can be efficiently coupled to the rest of a network [1-3]. While some of the basic functionalities are presently available in the form of individual components, their integration is still challenging for a number of reasons, including their size, speed, and power consumption. Therefore, the development of optoplasmonic devices that could be integrated on a single chip and could bridge existing gaps in optoelectronic integration is of paramount importance.

One of the major requirements for the realization of efficiently performing optoelectronics circuits is the ability to buffer optical signals so that the data traffic jams are prevented. An optical buffer is a device that slows down (or even stops) light to store it for a certain period of time. Although several approaches to the realization of such structures have been demonstrated, a majority of slow light schemes based on various waveguide geometries are not easily scalable to a chip-size footprint [1].

It is well known that optical bistability, a phenomenon in which two different values of output power are possible for the same input power, finds numerous applications in optical memory and storage devices. Therefore, realized in compact configuration, it can provide a viable solution for all-optical on-chip storage applications.

Metamaterials (MMs) were shown to enable subwavelength waveguides and cavities - a property that fundamentally differentiates them from conventional-materialsbased light wave components $[4,5]$. Therefore, in this work, we investigate the most general solution for wave interactions in positive-negative index MM-based nonlinear optical couplers (shown in Figure 1). It should be mentioned that nonlinear optical couplers made of conventional positive index materials (PIMs) are not bistable (unless some additional components providing optical feedback are introduced) [6]. However, in MM-based couplers, bistability results from the effective feedback mechanism enabled by the opposing directionality of phase and energy velocities in negative index materials (NIMs). Moreover, such a coupler supports gap solitons - a feature commonly associated with periodic structures $[7,8]$. These unusual properties of MM directional couplers form a basis for the development of all-optical processing applications, including wavelength converters, flip-flops, and mirrorless lasers.

Our previous studies focused on particular cases of phase-matched symmetric couplers with identical nonlinear properties and on asymmetric couplers with only one nonlinear channel. In this work, we found a generalized analytical solution in the presence of phase mismatch and for arbitrary values of nonlinear coefficients of both channels. 

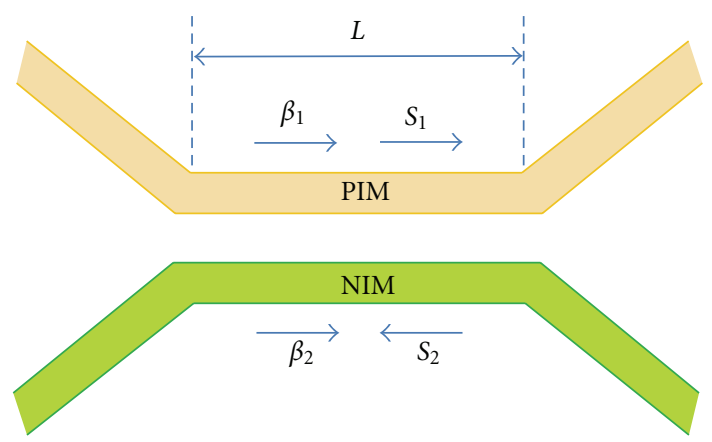

Figure 1: Positive-negative index nonlinear optical coupler.

The availability of such a solution enables novel optimized designs of such couplers.

\section{Theoretical Model}

As it has been mentioned above, in this work, the case of continuous wave propagation in a directional PIM-NIM coupler with third-order Kerr nonlinearity [9] is considered. From Maxwell's equations in a slowly varying envelope approximation the following system of differential equations, which can describe continuous wave propagation in such optical system, can be derived $[9,10]$ :

$$
\begin{gathered}
i \sigma_{1} \frac{\partial a_{1}}{\partial z}+\kappa_{12} a_{2} \exp (-i \delta z)+\gamma_{1}\left|a_{1}\right|^{2} a_{1}=0 \\
i \sigma_{2} \frac{\partial a_{2}}{\partial z}+\kappa_{21} a_{1} \exp (i \delta z)+\gamma_{2}\left|a_{2}\right|^{2} a_{2}=0
\end{gathered}
$$

here $a_{1}$ and $a_{2}$ are the complex amplitudes of the waves in the PIM and NIM channels, respectively, $\kappa_{12}$ and $\kappa_{21}$ are coupling coefficients, $\gamma_{1}$ and $\gamma_{2}$ are normalized nonlinearity coefficients, $\delta=\beta_{1}-\beta_{2}$ is the mismatch between the propagation constants in the channels, and $\sigma_{i}$ is the sign of the refractive index. In the case of the PIM-NIM coupler $\sigma_{1}>0, \sigma_{2}<0$. It is worth noting that the magnitude of the coupling coefficients depends on the geometrical parameters, such as channel width and separation distance between the channels, as well as on the channels dielectric properties. In a particular situation, it may be difficult to fabricate two identical waveguides with different signs of the refraction indexes, but, without losing any generalization of the analytical solution, it can be assumed that coupling coefficients of both channels are equal.

Let us represent complex amplitudes $a_{1}$ and $a_{2}$ in terms of real amplitudes $A_{i}$ and phases $\phi_{i}$ :

$$
a_{1}=A_{1} \exp \left(i \phi_{1}\right), \quad a_{2}=A_{2} \exp \left(i \phi_{2}\right) .
$$

Substituting (2) into (1) and separating the real and imaginary parts lead to the following system of equations:

$$
\begin{gathered}
\frac{\partial A_{1}}{\partial z}=\kappa_{12} A_{2} \sin (\theta), \\
\frac{\partial A_{2}}{\partial z}=\kappa_{21} A_{1} \sin (\theta), \\
\frac{\partial \theta}{\partial z}=\left(\kappa_{12} \frac{A_{2}}{A_{1}}+\kappa_{21} \frac{A_{1}}{A_{2}}\right) \cos (\theta)+\gamma_{1} A_{1}^{2}+\gamma_{2} A_{2}^{2}+\delta,
\end{gathered}
$$

here $\theta=\phi_{1}-\phi_{2}+\delta z$.
If the light is initially launched into channel 1 , then the boundary conditions take the following form:

$$
A_{1}(0)=A_{0}, \quad A_{2}(L)=0,
$$

where $A_{0}$ is the amplitude of the input signal and $L$ is the length of the coupler. Assuming that coupling coefficients $\kappa_{12}=\kappa_{21}=\kappa$, we can rewrite (3) as follows:

$$
\begin{gathered}
\frac{\partial A_{1}}{\partial z}=\kappa A_{2} \sin (\theta), \\
\frac{\partial A_{2}}{\partial z}=\kappa A_{1} \sin (\theta), \\
\frac{\partial \theta}{\partial z}=\kappa\left(\frac{A_{2}}{A_{1}}+\frac{A_{1}}{A_{2}}\right) \cos (\theta)+\gamma_{1} A_{1}^{2}+\gamma_{2} A_{2}^{2}+\delta .
\end{gathered}
$$

Next, we found that (5) have two integrals of motion:

$$
\begin{gathered}
P_{1}-P_{2}=C, \\
\Gamma=A_{2}^{2}\left[\left(\gamma_{2}-\gamma_{1}\right) A_{2}^{2}+2 \gamma_{1} A_{1}^{2}+2 \delta\right]+4 \kappa A_{1} A_{2} \cos (\theta),
\end{gathered}
$$

where $P_{1}=A_{1}^{2}$ and $P_{2}=A_{2}^{2}$. From the boundary conditions (4) one can conclude that $C=A_{1}^{2}(L)$ and $\Gamma=0$. From these integrals of motion, we can derive an expression for $\cos (\theta)$ :

$$
\cos (\theta)=-\frac{A_{2}^{2}\left(\left(\gamma_{1}+\gamma_{2}\right) A_{2}^{2}+2\left(\delta+\gamma_{1} C\right)\right)}{4 \kappa A_{1} A_{2}} .
$$

Substituting (7) into the second equation of (5), we derive an equation for power evolution in the second channel:

$$
\left(\frac{\partial P_{2}}{\partial z}\right)^{2}=4 \kappa^{2} P_{1} P_{2}-P_{2}^{2}\left(\tilde{\gamma} P_{2}+\tilde{\delta}\right)
$$

where $\tilde{\gamma}=\left(\gamma_{1}+\gamma_{2}\right) / 2$ and $\tilde{\delta}=\delta+\gamma_{1} C$. Using the first integral of motion, we can rewrite this equation in terms of $P_{2}$ :

$$
\left(\frac{\partial P_{2}}{\partial z}\right)^{2}=-\tilde{\gamma} P_{2}^{4}-2 \tilde{\gamma} \tilde{\delta} P_{2}^{3}+\left(4 \kappa^{2}-\tilde{\delta}\right) P_{2}^{2}+4 \kappa^{2} C P_{2} .
$$

The solutions for $P_{1}$ and $P_{2}$ are found in terms of the Weierstrass elliptic function $\wp\left(z ; g_{2}, g_{3}\right)$ :

$$
\begin{aligned}
& P_{1}(z)=C+\frac{\kappa^{2} C}{\left(\wp\left(L-z ; g_{2}, g_{3}\right)-(1 / 12)\left(4 \kappa^{2}-\widetilde{\delta}^{2}\right)\right)}, \\
& P_{2}(z)=\frac{\kappa^{2} C}{\left(\wp\left(L-z ; g_{2}, g_{3}\right)-(1 / 12)\left(4 \kappa^{2}-\widetilde{\delta}^{2}\right)\right)} .
\end{aligned}
$$

The invariants $g_{2}$ and $g_{3}$ are defined as follows:

$$
\begin{gathered}
g_{2}=2 \tilde{\gamma} \tilde{\delta} \kappa^{2} C+\frac{\left(4 \kappa^{2}-\tilde{\delta}\right)^{2}}{12} \\
g_{3}=-\frac{\tilde{\gamma} \tilde{\delta} \kappa^{2} C\left(4 \kappa^{2}-\tilde{\delta}\right)}{6}-\left(\frac{4 \kappa^{2}-\tilde{\delta}}{6}\right)^{3}+\tilde{\gamma} \kappa^{4} C^{2} .
\end{gathered}
$$

The parameter $C$ can be found by solving the following transcendental equation:

$$
A_{0}^{2}=C+\frac{\kappa^{2} C}{\left(\wp\left(L ; g_{2}, g_{3}\right)-(1 / 12)\left(4 \kappa^{2}-\tilde{\delta}^{2}\right)\right)} .
$$


Since this is the most general solution for PIM-NIM couplers, first, we test it against previously considered particular cases $[7,8,10]$ : (i) a linear phase-matched coupler with $\gamma_{1}=\gamma_{2}=\delta=0$, (ii) a nonlinear phase-matched coupler with $\gamma_{1}=\gamma_{2}=\gamma, \delta=0$, and (iii) phase-matched asymmetric couplers. In the first case, the discriminant of the Weierstrass function $\Delta=g_{2}^{3}-27 g_{3}^{2}=0$. Therefore, in this case we can rewrite (10) in terms of hyperbolic functions. So we get the following solution for the linear case of a PIM-NIM coupler [9]:

$$
\begin{gathered}
P_{1}(z)=C\left(1+\sinh (\kappa(L-z))^{2}\right), \\
P_{1}(z)=C \sinh (\kappa(L-z))^{2} .
\end{gathered}
$$

In the second case, by expanding the Weierstrass function $\wp\left(z ; g_{2}, g_{3}\right)$ in terms of the Jacobi elliptic function one can derive the same expressions for $P_{1}(z)$ and $P_{2}(z)$ as in [7].

Next, we compare two asymmetric cases: (a) a phasematched coupler in which the PIM channel is nonlinear and the NIM channel is linear, and (b) a phase-matched coupler in which the PIM channel is linear and the NIM channel is nonlinear. Figure 2 shows a numerical solution of (12), which determines the dependence of the output signal power $P_{1}(L)$ on the input field intensity $P_{1}(0)$, where $\kappa=8, \delta=0$, and $L=1$. The solid, black curve represents the case when $\gamma_{1}=5, \gamma_{2}=0$, that is, case (a); the blue, dashed curve shows the opposite case when $\gamma_{1}=0, \gamma_{2}=5$, that is, case (b).

These results show in both limiting cases that the phasematched PIM-NIM coupler is bi- (or multi-) stable. Also, it is noteworthy that for given combinations of the linear coupling coefficient and the length of the coupler, the bistability threshold is higher only in the second (NIM) channel, which is nonlinear while light initially is pumped into the first (PIM) channel. This is due to the fact, that in this case, nonlinearity takes effect only if enough power is coupled to the second (nonlinear) channel.

\section{Effect of Phase Mismatch}

So far, we have considered the behavior of somewhat idealized PIM-NIM couplers-phase-matched couplers with $\delta=0$. However, from a practical realization viewpoint, it would be important to analyze the influence of the phase mismatch during wave propagation in such systems. In this section, we investigate the dependence of $P_{1}(z)$ and $P_{2}(z)$ on $\delta$.

Figure 3 represents the output power $P_{1}(L)$ as a function of input power $P_{1}(0)$ when $\kappa=8, \gamma_{1}=5$ and $\gamma=0$ (selffocusing nonlinearity only in the first channel) with different values of phase mismatch $\delta$ : solid black curve: $\delta=0$; blue curve; $\delta=10$; dashed red curve: $\delta=-10$. It is noteworthy that the dependence of the output field intensity $P_{1}(L)$ on input field power $P_{1}(0)$ depends on the sign of phase mismatch $\delta$. This can be understood by considering the dispersion relation for this system.

Assuming that the solution of (1) has the following form:

$$
a_{1,2}=u_{1,2} \exp (i q z) \exp \left( \pm i \frac{\delta}{2} z\right)
$$

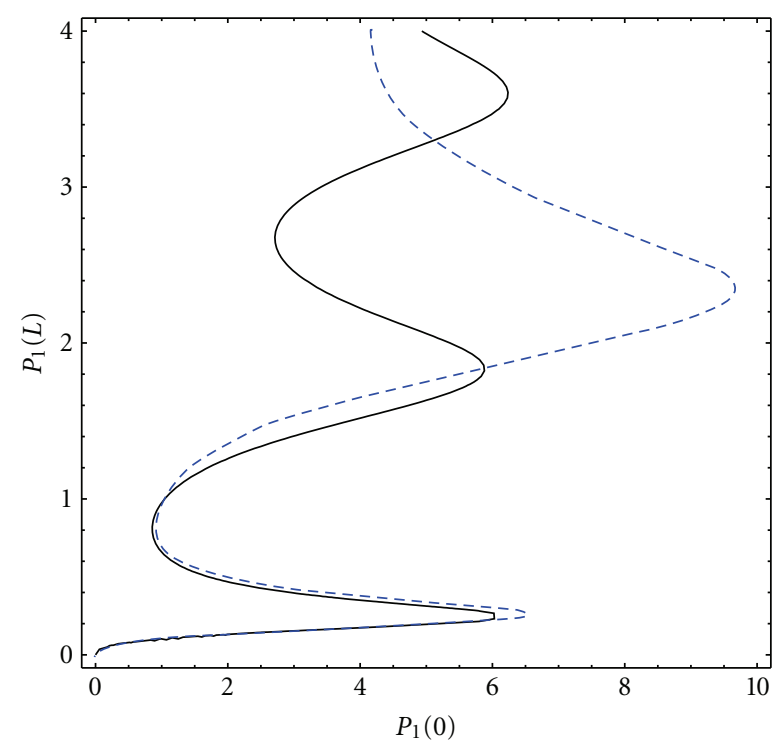

FIgure 2: Output power $P_{1}(L)$ as a function of input power $P_{1}(0)$ when $\kappa=8$ and $\delta=0$. Solid black curve: $\gamma_{1}=5, \gamma_{2}=0$; dashed blue curve: $\gamma_{1}=0, \gamma_{2}=5$.

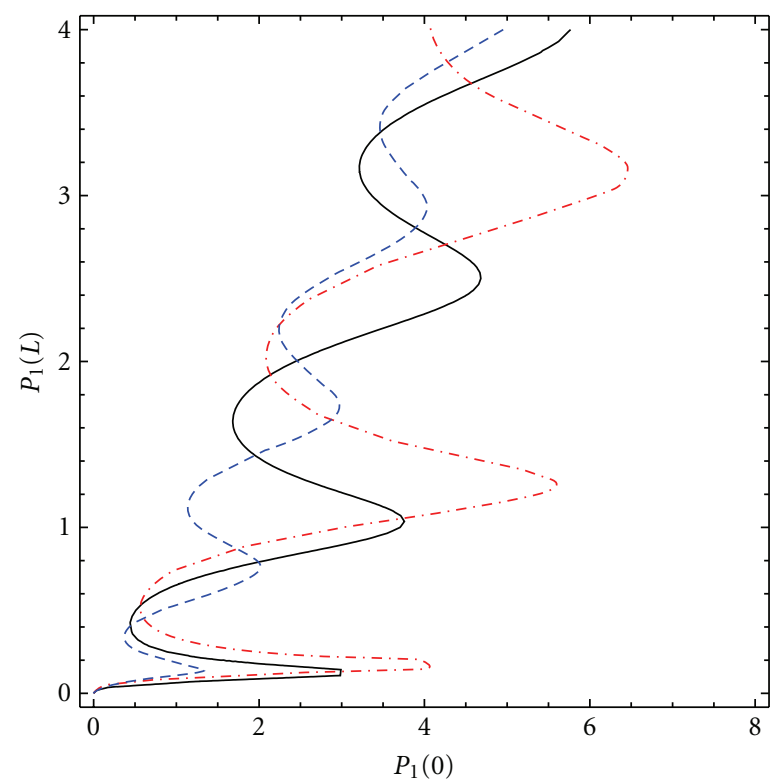

Figure 3: Output power $P_{1}(L)$ as a function of input power $P_{1}(0)$ when $\kappa=8, \gamma_{1}=\gamma_{2}=5$ (self-focusing nonlinearity). Solid black curve: $\delta=0$; dashed blue curve: $\delta=10$; dot-dashed red curve $\delta=$ -10 .

we can find that $\delta$ and $q$ have the following forms:

$$
\begin{aligned}
& \delta=-\frac{\kappa_{21}+\kappa_{12}\left(1-C / P_{0}\right)}{ \pm \sqrt{1-C / P_{0}}}-P_{0}\left(\gamma_{1}+\gamma_{2}\left(1-\frac{C}{P_{0}}\right)\right), \\
& q=-\frac{\kappa_{21}-\kappa_{12}\left(1-C / P_{0}\right)}{ \pm 2 \sqrt{1-C / P_{0}}}-P_{0}\left(\gamma_{2}\left(1-\frac{C}{P_{0}}\right)-\gamma_{1}\right),
\end{aligned}
$$

where because of the boundary conditions $C \in\left[0, P_{0}\right]$. 


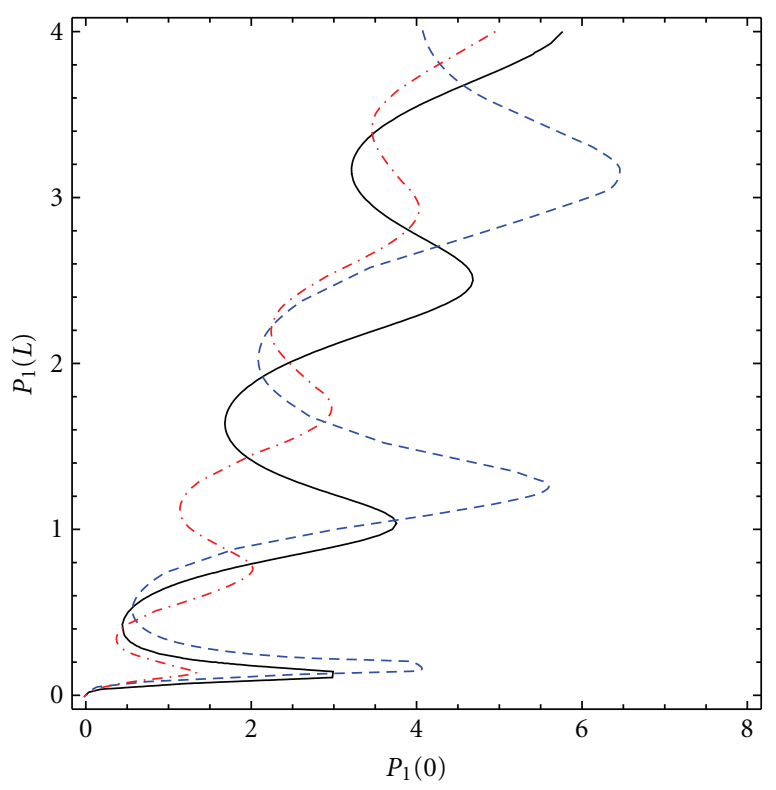

FIgURe 4: Output power $P_{1}(L)$ as a function of input power $P_{1}(0)$ when $\kappa=8, \gamma_{1}=\gamma_{2}=-5$ (self-defocusing nonlinearity). Solid black curve: $\delta=0$; dashed blue curve: $\delta=10$; dot-dashed red curve $\delta=-10$.

In a linear PIM-NIM coupler, these dispersion relations show a bandgap [10]. Let us fix two values of detuning to be $\delta_{+,-}= \pm \delta_{0}$ in the linear case such that the center of the bandgap corresponds to $\delta=0$. Next, from (15) we find that in the case of positive nonlinear coefficients (selffocusing Kerr nonlinearity) the " $\delta-q$ " dispersion relations shift towards negative values of $\delta$ leading to asymmetry of the center of the bandgap with respect to $\delta_{+}$and $\delta_{-}$and, as a result, different dependences of the output field intensity on input field power on either side of the bandgap [7]. In contrast, in the case of negative nonlinear coefficients (selfdefocusing Kerr nonlinearity) the " $\delta-q$ " dispersion relations shift towards positive values of $\delta$ and the dependences of the output field intensity on input field power reverse. Indeed, if now we plot the output power $P_{1}(L)$ as a function of input power $P_{1}(0)$ when $\kappa=8$ and $\gamma_{1}=\gamma_{2}=-5$ (self-defocusing nonlinearity only in the first channel) with different values of phase mismatch $\delta$ : solid black curve: $\delta=0$; blue curve: $\delta=10$; dashed red curve: $\delta=-10$ (see Figure 4), we find that the curves corresponding to positive and negative detuning interchanged as compared to those in Figure 3.

\section{Discussion}

Although the analysis of the PIM-NIM coupler in this work was based on coupled-mode equations assuming effective medium parameters for the dielectric permittivity and magnetic permeability of MMs, in practice such an NIM channel (which is the most challenging part of the proposed device) can be realized in at least two configurations: (i) using double-negative resonant MMs and (ii) using strongly anisotropic MM waveguides. Such waveguides were shown to support negative-index propagating modes $[11,12]$. For these modes, the wave propagation is in a direction opposite to the phase velocity. As a result, the waveguide behaves as a 2-dimensional counterpart of 3-dimensional negative index material. Such a waveguide can be designed using alternating metal and dielectric subwavelength layers with positive and negative permittivities, respectively. As a nonlinear optical material, we envision incorporate chalcogenide glasses or nonlinear polymers that possess relatively high nonlinear refractive indices. Theoretical design and experimental realization of such devices will be discussed elsewhere.

In conclusion, we found a generalized analytical solution for nonlinear wave interactions in PIM-NIM couplers in the presence of phase mismatch and for arbitrary values of nonlinear coefficients in both channels. These results offer a practical tool for designing novel MM-based couplers based on either double-negative or strongly anisotropic MMs that are likely to enable ultracompact optical storage and memory components for photonics on chip applications.

\section{Acknowledgment}

This work was supported by the US Army Research Office Award no. W911NF0910075.

\section{References}

[1] Yu. A. Vlasov, M. O’Boyle, H. F. Hamann, and S. J. McNab, "Active control of slow light on a chip with photonic crystal waveguides," Nature, vol. 438, no. 7064, pp. 65-69, 2005.

[2] Yu. Vlasov, W. M. J. Green, and F. Xia, "High-throughput silicon nanophotonic wavelength-insensitive switch for onchip optical networks," Nature Photonics, vol. 2, no. 4, pp. 242246, 2008.

[3] L. Liu, R. Kumar, K. Huybrechts et al., "An ultra-small, lowpower, all-optical flip-flop memory on a silicon chip," Nature Photonics, vol. 4, no. 3, pp. 182-187, 2010.

[4] N. Engheta and R. W. Ziolkowski, Metamaterials: Physics and Engineering Explorations, John Wiley \& Sons, 2006.

[5] K. L. Tsakmakidis, A. D. Boardman, and O. Hess, "'Trapped rainbow' storage of light in metamaterials," Nature, vol. 450, no. 7168, pp. 397-401, 2007.

[6] S. M. Jensen, "The nonlinear coherent coupler," IEEE Journal of Quantum Electron, vol. 18, no. 10, pp. 1580-1583, 1982.

[7] N. M. Litchinitser, I. R. Gabitov, and A. I. Maimistov, "Optical bistability in a nonlinear optical coupler with a negative index channel," Physical Review Letters, vol. 99, no. 11, Article ID 113902, 4 pages, 2007.

[8] G. Venugopal, Z. Kudyshev, and N. M. Litchinitser, "Asymmetric positive-negative index nonlinear waveguide couplers," IEEE Journal of Selected Topics in Quantum Electronics, vol. 17, pp. 1-4, 2007.

[9] G. P. Agrawal, Nonlinear Fiber Optics, Academic Press, 2007.

[10] A. Alu and N. Engheta, Negative-Refraction Metamaterials, John Wiley \& Sons, New York, NY, USA, 2005, edited by G. V. Eleftheriades and K. G. Balmain.

[11] V. A. Podolskiy and E. E. Narimanov, "Strongly anisotropic waveguide as a nonmagnetic left-handed system," Physical Review B, vol. 71, no. 20, Article ID 201101, 4 pages, 2005.

[12] A. J. Hoffman, V. A. Podolskiy, D. L. Sivco, and C. Gmachl, "Sub-diffraction negative and positive index modes in midinfrared waveguides," Optics Express, vol. 16, no. 21, pp. 16404-16409, 2008. 

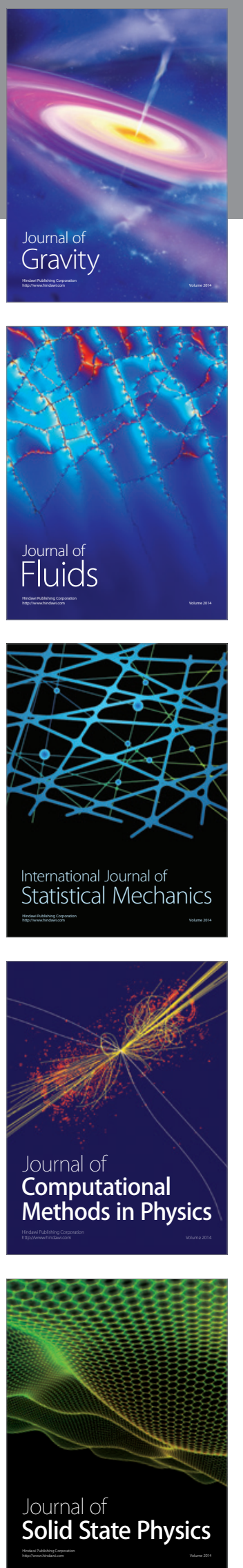

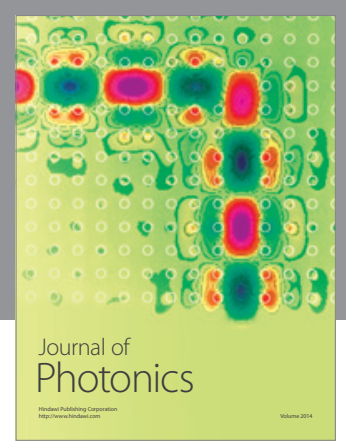

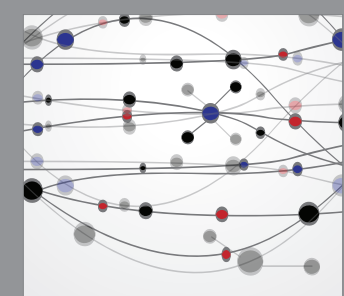

The Scientific World Journal
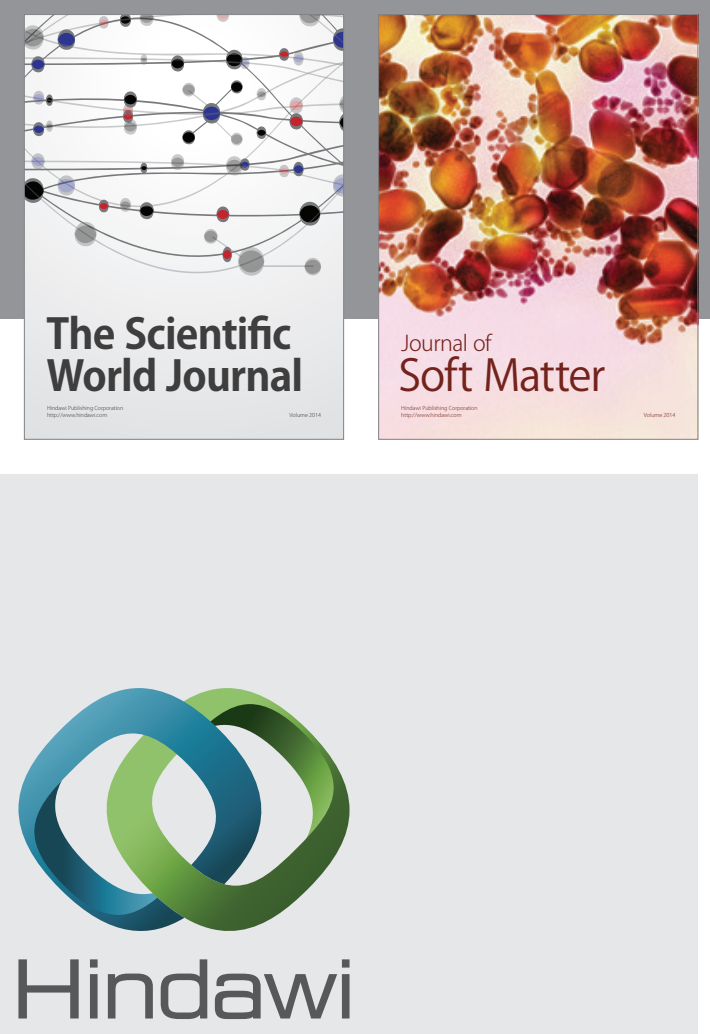

Submit your manuscripts at

http://www.hindawi.com
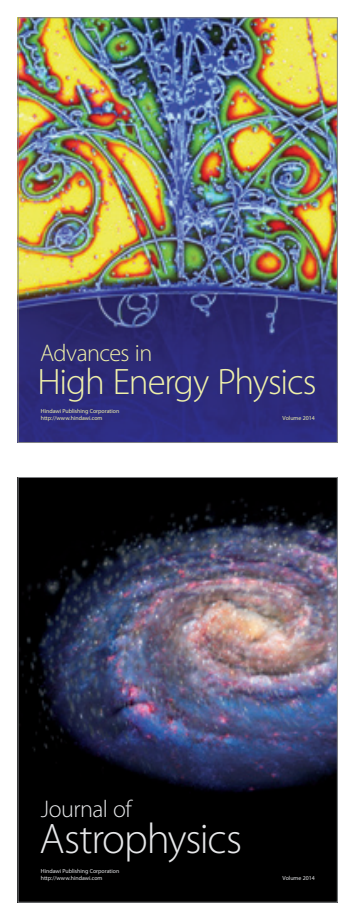
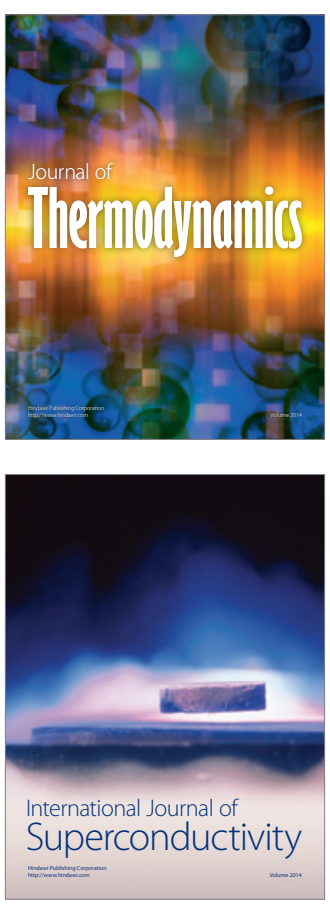
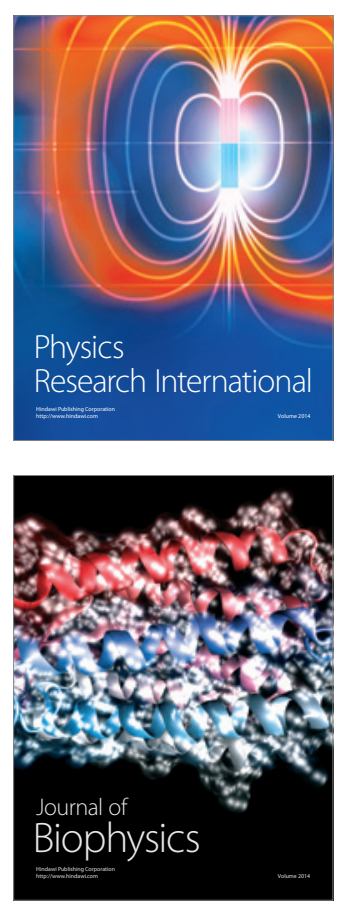
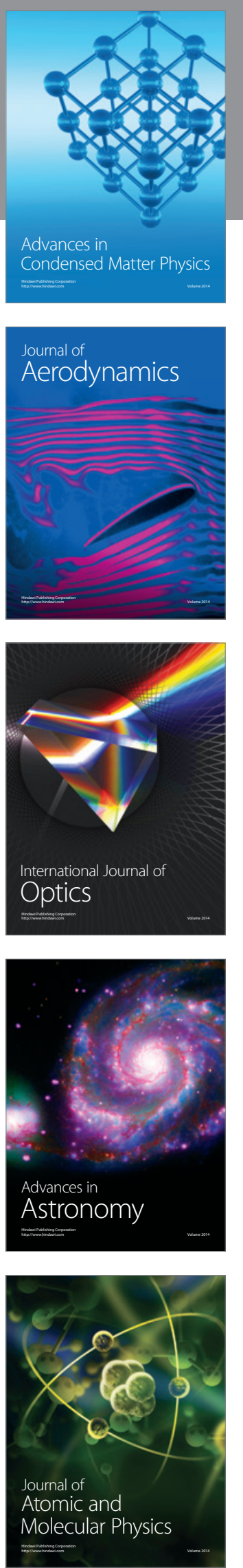